\title{
Stent-Assisted Coiling of Wide-Neck Intracranial Aneurysms Using Low-Profile LEO Baby Stents: Initial and Midterm Results
}

\author{
K. Aydin, A. Arat, S. Sencer, M. Barburoglu, and S. Men
}

\begin{abstract}
BACKGROUND AND PURPOSE: Low-profile self-expandable stents were recently introduced for the treatment of wide-neck intracranial aneurysms. This study investigated the initial and midterm clinical and angiographic results of LEO Baby stent-assisted coiling in the treatment of wide-neck intracranial aneurysms.
\end{abstract}

MATERIALS AND METHODS: A retrospective review was performed to identify patients who were treated with LEO Baby stent-assisted coiling. Eighty patients with 80 wide-neck intracranial aneurysms were included in the study. Eleven patients (13.8\%) presented with subarachnoid hemorrhage. All patients were treated with LEO Baby stent-assisted coiling. Technical success and immediate postprocedural clinical and angiographic outcomes were evaluated. Seventy-three patients attended angiographic and clinical follow-up for a mean duration of $7.2 \pm 3.8$ months. Periprocedural and delayed complications were reviewed. Preprocedural and follow-up clinical statuses were assessed by using the modified Rankin Scale.

RESULTS: The technical success rate of the procedure was $97.5 \%$. The immediate postprocedural angiography revealed a complete occlusion of the aneurysm in $75 \%$ of the 80 patients. The last follow-up angiograms showed complete occlusion in $85.7 \%$ of the 77 patients with an angiographic follow-up. Of the 77 patients with a follow-up angiography, $6.5 \%$ showed an increase in the filling status of the aneurysm and $5.2 \%$ required retreatment. The overall procedure-related complication rate, including asymptomatic complications, was $11.3 \%$. The permanent morbidity rate was $3.8 \%$. There was no mortality in this study.

CONCLUSIONS: This case series demonstrates the relative safety, efficacy, and midterm durability of the LEO Baby stent-assisted coiling procedure for the treatment of wide-neck intracranial aneurysms.

ABBREVIATIONS: AcomA = anterior communicating artery; $\mathrm{SAC}=$ stent-assisted coiling

C iling has become the most common treatment for intracranial aneurysms since the results of the International Subarachnoid Aneurysm study were published. ${ }^{1}$ However, treatment of wide-neck complex aneurysms remains challenging. The relatively high recurrence rates after coiling are another major limitation of the endovascular treatment of wide-neck and/or large aneurysms. ${ }^{2,3}$ The stent-assisted coiling (SAC) technique was developed to treat wide-neck intracranial aneurysms. ${ }^{4}$ Several selfexpandable stents dedicated to intracranial use were introduced

Received January 12, 2015; accepted after revision February 25.

From the Department of Radiology (K.A., S.S., M.B.), Neuroradiology Division, Istanbul Faculty of Medicine, Istanbul University, Istanbul, Turkey; Department of Radiology (A.A.), School of Medicine, Hacettepe University, Ankara, Turkey; and Department of Radiology (S.M.), Dokuz Eylul University, Izmir, Turkey.

Please address correspondence to Kubilay Aydin, MD, Istanbul University, Istanbul Faculty of Medicine, Department of Radiology, Neuroradiology Division, Istanbul 34340, Turkey; e-mail: dr.aydink@superonline.com

- Indicates open access to non-subscribers at www.ajnr.org

http://dx.doi.org/10.3174/ajnr.A4355 in the past decade, and the availability of these self-expandable intracranial stents enabled the endovascular treatment of wideneck complex aneurysms that were uncoilable previously., Stents create a mechanical scaffold that prevents coil protrusion into the parent artery. Stents also reduce the risk of recanalization because of their hemodynamic and biologic effects. ${ }^{6}$

Low-profile self-expandable intracranial stents (LEO Baby; Balt, Montmorency, France; and LVIS Jr; MicroVention, Tustin, California) were introduced recently. ${ }^{7,8}$ The designs of these stents enable their delivery into small arteries. These low-profile intracranial stents can be deployed into arteries with diameters as small as $1.5 \mathrm{~mm}$ to treat wide-neck aneurysms. Furthermore, these stents can be delivered through microcatheters with an internal diameter of 0.0165 inches, which facilitates navigation in small-sized vessels.

This multicenter retrospective study investigated the safety and efficacy of LEO Baby stent-assisted coiling treatment of wideneck intracranial aneurysms. We also evaluated the midterm (6-12 months) durability of LEO Baby SAC treatment. 


\section{MATERIALS AND METHODS}

\section{Patients}

After approval of the study by the institutional review board, a retrospective review of interventional data base records of 3 university hospitals was performed to identify patients who were treated by using at least 1 LEO Baby stent between October 2012 and November 2014. Medical records and radiologic images of patients were gathered. Three neuroradiologists (K.A., M.B., and S.M.) assessed the surgery reports, medical charts, and radiologic images of the patients. Patient demographics and presenting symptoms, the location and size of aneurysms, previous treatment history, stent-deployment success, technical and clinical complications, and the degree of aneurysm occlusion were recorded.

Eighty-six patients received attempted treatment by using LEO Baby stents between October 2012 and November 2014. Only patients treated by using the SAC technique were included into the study; 6 patients who were treated by using stent monotherapy without coiling were excluded. LEO Baby stent-assisted coiling was performed in the treatment of aneurysms located in the parent arteries with a diameter of $\leq 3.5 \mathrm{~mm}$ and with a dome/ neck ratio of $<2$ or a neck diameter of $>4 \mathrm{~mm}$, a morphology not suitable for primary coiling.

This study consisted of 80 patients with 80 aneurysms. Fifty of these aneurysms were treated in (Hacettepe University Medical School Hospital), 23 in (Istanbul Medical School Hospital), and 7 in (Dokuz Eylul University Hospital). There were 31 male and 49 female $(61 \%)$ patients, with a mean age of $52.05 \pm 10.17$ years (range, $26-73$ years). Eleven patients $(13.8 \%)$ presented with subarachnoid hemorrhage in a subacute (2-4 weeks) phase, and the remaining 69 patients $(86.2 \%)$ had unruptured aneurysms. No patient was stented during the acute phase of subarachnoid hemorrhage ( $<2$ weeks). Seventy-two of 80 aneurysms (90\%) were incidental and had not received prior treatment. Eight aneurysms (10\%) were recanalized aneurysms that were treated previously by using conventional coiling, balloon-assisted coiling, or open surgery (clipping). The mean dome size of the aneurysms was $7.26 \pm 3.41 \mathrm{~mm}$ (range, 3-22 $\mathrm{mm}$ ). The mean neck size was $4.44 \pm 1.18 \mathrm{~mm}$ (range, $2.5-9 \mathrm{~mm}$ ). The aneurysms were located in the middle cerebral artery bifurcation in 43 patients $(53.8 \%)$, the anterior communicating artery (AcomA) in 27 patients (33.8\%), the pericallosal artery in 1 patient $(1.2 \%)$, the internal carotid artery bifurcation in 1 patient $(1.2 \%)$, the basilar artery bifurcation/vertebrobasilar junction in 7 patients $(8.6 \%)$, and the posterior inferior cerebellar artery in 1 patient $(1.2 \%)$. The mean parent artery diameter was $2.35 \pm 0.43 \mathrm{~mm}$ (range, $1.3-3.5 \mathrm{~mm}$ ).

Preoperative patient conditions were evaluated by using the modified Rankin Scale. The preoperative mRS scores of all patients except 1 were 0 . One patient was assigned a preprocedural mRS score of 3 because of a previous history of subarachnoid hemorrhage.

\section{General Description of the Endovascular Procedure}

Patients received $75 \mathrm{mg}$ clopidogrel and $300 \mathrm{mg}$ aspirin daily for at least 5 days before the procedure. Clopidogrel resistance was evaluated before the procedures (VerifyNow P2Y12 assay; Accumetrics, San Diego, California) to ensure a good response (platelet

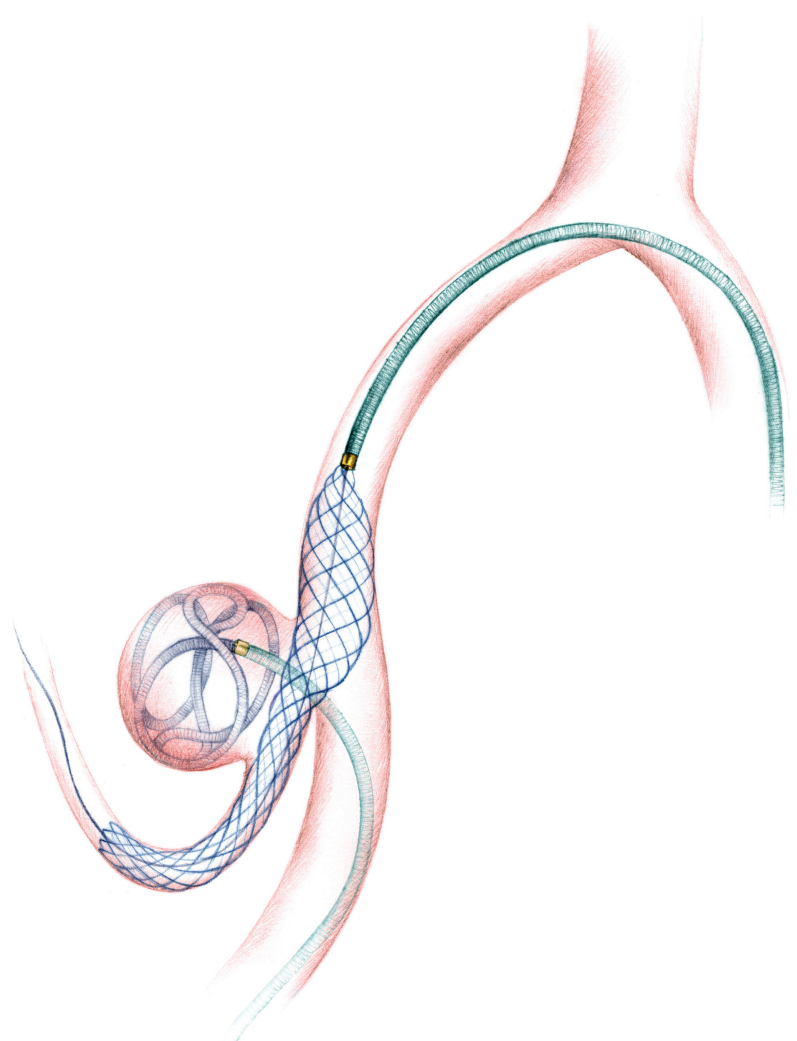

FIG 1. An illustration showing the LEO Baby stent-assisted coiling treatment of a PICA aneurysm. A microcatheter is jailed into the sac of aneurysm, while the LEO Baby stent is deployed to the parent artery through a second microcatheter.

aggregation inhibition $>40 \%$ ) to clopidogrel. Every endovascular procedure was performed by using a femoral approach with the patient under general anesthesia. Systemic anticoagulation was initiated immediately after the insertion of a femoral introducer sheath with a bolus dose of 5000 IU of IV heparin. The bolus dose was followed by a slow heparin infusion to maintain an activated clotting time of approximately twice the baseline value. A 6F guiding catheter or sheath was placed in the target artery. In patients with tortuous vascular anatomy, a distal access catheter (Fargomax; Balt; or Neuron 070; Penumbra, Alameda, California) was navigated through the guiding sheath into the petrous segment of the internal carotid artery or to the V2 segment of the vertebral artery. Aneurysm sacs were catheterized by using an Excelsior SL-10 (Stryker Neurovascular, Kalamazoo, Michigan) or Headway 17 (MicroVention) microcatheter with a soft-tipped 0.014inch microguidewire. A second microcatheter (Excelsior SL-10, Headway 17, or Vasco10; Balt) was navigated across the aneurysm for stent placement (Fig 1).

The same microcatheter was used for stent placement and coiling if the jailing technique was not performed. Stent sizes were chosen on the basis of the largest diameter of the parent artery and the length of the aneurysm neck. Coiling was performed by using bare platinum detachable coils after the stents were deployed. Aneurysms were coiled until complete occlusion was achieved or no further coils could be safely deployed within the aneurysm sac. Femoral puncture sites were sealed by using an arterial closure device. Postprocedural dual antiplatelet treatment, including 75 
$\mathrm{mg}$ /day of clopidogrel and $300 \mathrm{mg}$ /day of aspirin, was continued for 3 months. Dual antiplatelet therapy was switched to aspirin thereafter. Aspirin was continued life-long unless a contraindication existed. Dual antiplatelet treatment was prolonged to at least 6 months in patients who received $>1$ stent.

\section{Stent-Placement Configurations}

Sixty-six of 80 aneurysms (82.5\%) were coiled with the assistance of a single LEO Baby stent. Fourteen aneurysms (17.5\%) were coiled with the assistance of 2 stents in various configurations. Enterprise (Codman \& Shurtleff, Raynham, Massachusetts) and LEO (Balt) stents were used as the first stent in the treatment of 3 aneurysms followed by the placement of a LEO Baby stent as the second stent. In the double LEO Baby cases, 6 aneurysms were treated by using Y-stent placement and 2 aneurysms received temporary Y-stent placement. T, X, and parallel stent placements were performed for the treatment of 8 aneurysms. Enterprise and LEO Baby stents were used as a bailout stent placement in 2 patients to treat complications.

\section{Follow-Up}

Immediate postprocedural angiograms were obtained to evaluate aneurysm occlusion according to the Raymond classification. ${ }^{9}$ Endovascular treatment was considered successful when stent deployment was successful and adequate angiographic occlusion by using coiling was achieved. The development of any technical complication was considered a technical failure, regardless of angiographic and clinical outcomes. Technical and clinical complications were recorded. Complications were "periprocedural" if they occurred within 48 hours postprocedure or "delayed" if they occurred later than 48 hours postprocedure. Postprocedural control cranial CT examinations were performed within 12 hours following the endovascular procedures. The first angiographic follow-up was performed at 3-6 months by using either MR angiography or conventional angiography, depending on the results of the endovascular procedure. The follow-up MR angiography examination included a 3D time-of-flight sequence performed before and after contrast material injection. The second and third angiographic follow-up DSAs were generally performed at 8-12 months and 18-24 months, respectively. Follow-up angiograms were assessed for the filling status of aneurysms and the development of in-stent stenosis or thrombosis. Progressive thrombosis on follow-up imaging was defined as an improvement in the Raymond class from sac or neck filling (class 3 or 2) toward total occlusion (class 1). Recanalization was defined as a deterioration in the Raymond class. Patient neurologic status was evaluated during discharge and during angiographic follow-ups by using the mRS score.

\section{RESULTS}

\section{Technical Success and Immediate Angiographic Results}

The endovascular procedure was successful in 78 of 80 patients (97.5\%) (Fig 2). Technical complications developed during LEO Baby stent deployment in 2 patients, but the angiographic and clinical outcomes of these patients were successful. Among the patients with successful procedures, the LEO Baby stent was successfully deployed on the first attempt in 77 patients and on the second attempt in 1 patient (Fig 3). Immediate postprocedural angiograms revealed a class 1 occlusion in 60 aneurysms (75\%), class 2 occlusion in 17 aneurysms (21.3\%), and class 3 occlusion in 3 aneurysms (3.7\%) (Fig 4).

\section{Complications}

Complications, including asymptomatic technical and periprocedural thromboembolic events, developed in 9 patients (11.3\%). Six patients $(7.5 \%)$ remained asymptomatic. However, complications resulted in a permanent morbidity in 3 patients $(3.8 \%)$. There was no mortality.

Technical complications without adverse clinical outcomes developed in 2 patients (2.5\%). Stent migration without clinical adverse events developed during deployment in 1 patient. In this patient, the LEO Baby stent migrated into the sac of the aneurysm, and 2 other stents were implanted to complete the coil embolization. In another patient, the control procedural angiography immediately after deployment of a LEO Baby stent revealed an incomplete apposition of the stent. Telescopic implantation of an Enterprise stent resulted in complete stent apposition.

Periprocedural or delayed thromboembolic events developed in 6 patients $(7.5 \%)$. Thromboembolic complications resulted in permanent neurologic deficits in 3 patients (3.8\%). Thromboembolic complications were periprocedural in 5 patients and delayed in 1 patient.

In-stent thrombus formation was visualized during the endovascular procedures in 4 patients. Two of these patients were treated by using an intra-arterial infusion of tirofiban (Aggrastat), and 2 other patients were treated by using a continuous intravenous infusion of tirofiban. An MCA infarct developed in 1 patient who was treated by using intra-arterial tirofiban. The MCA infarct in this patient resulted in dysphasia and hemiparesis. This patient's mRS score both at discharge and at the final follow-up was 3. The other 3 patients who developed in-stent thrombi during the procedures remained asymptomatic.

One patient presented with ipsilateral ischemic stroke 4 hours after treatment of a right MCA bifurcation aneurysm by using a single LEO Baby stent. Emergent control angiography revealed an acute in-stent thrombosis. A proximal malpositioning of the stent was also observed. Telescopic implantation of an Enterprise stent successfully secured arterial flow in this patient. However, the patient developed a basal ganglia infarction and presented with hemiparesis. The mRS score of this patient at discharge was 4 . This patient's symptoms resolved during several weeks, and her mRS score at the 6-month follow-up was 2 .

One patient presented with hemiparesis 3 months after a single LEO Baby SAC to treat an AcomA aneurysm. This patient's control angiography revealed complete in-stent thrombosis; his final follow-up mRS score was 3.

One hemorrhagic complication (1.3\%) developed during the treatment of an MCA bifurcation aneurysm. A mild extravasation from the aneurysm developed during coiling. Coiling was completed, and the patient awoke with a mild headache. The postprocedural cranial CT revealed that the contrast extravasation was confined to the Sylvian fissure. This patient did not develop any neurologic deficits. 

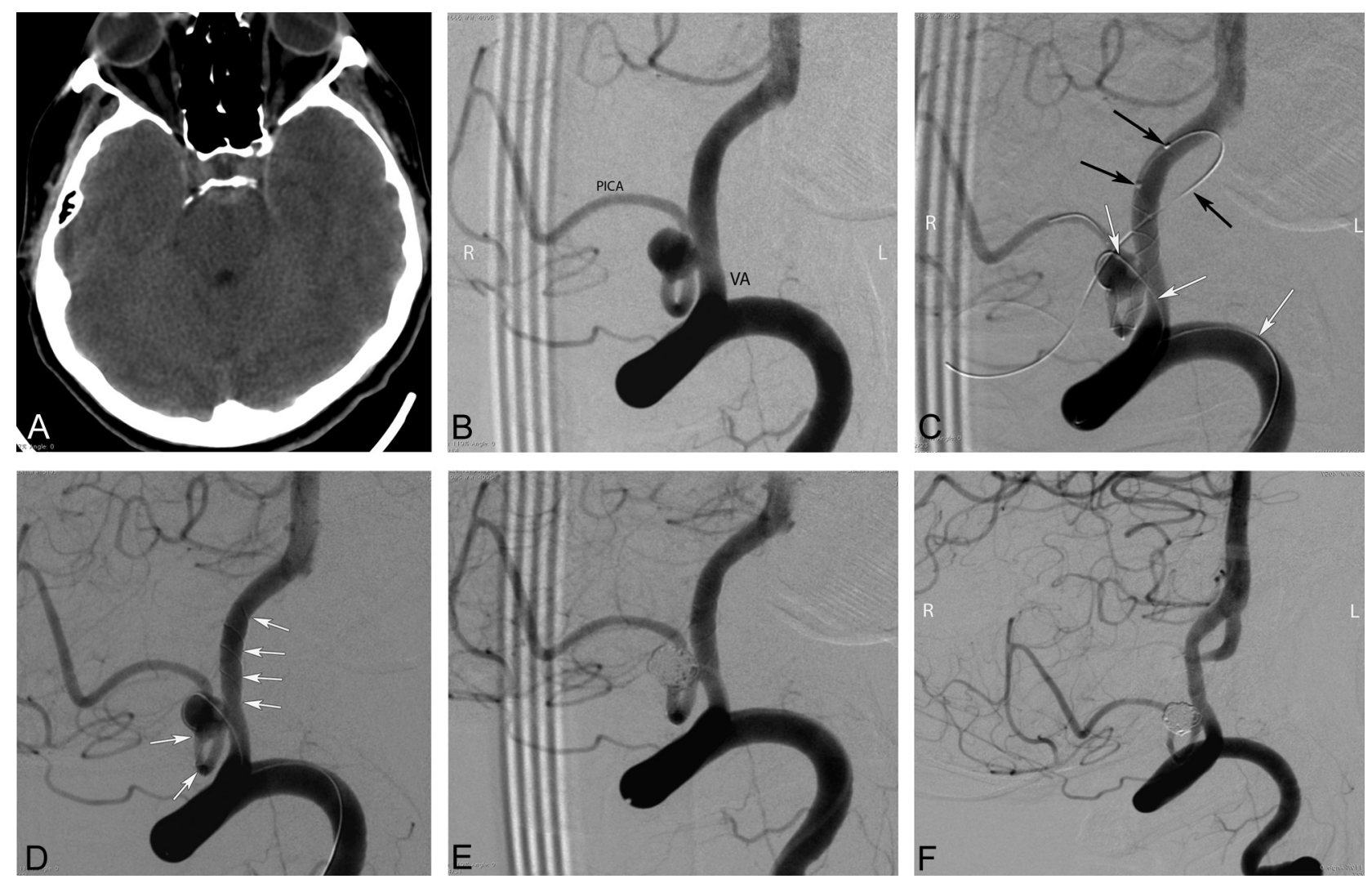

FIG 2. Preprocedural cranial CT and procedural and follow-up angiography images of a 44-year-old female patient with a ruptured PICA aneurysm. A, Cranial CT image obtained 9 days before the endovascular procedure ( 5 days following the rupture of aneurysm) shows the subarachnoid hemorrhage in the prepontine cistern. $B$, DSA image in a right anterior oblique projection reveals a 5-mm wide-neck aneurysm located on the medullary segment of the left PICA. C, DSA images obtained during the procedure show the catheterization of the parent artery for stent placement (black arrows) from the contralateral (right) vertebral artery and jailing of the coiling microcatheter (white arrows) through the ipsilateral (left) vertebral artery (the small filling defect in the aneurysm sac is a small air bubble introduced by the delivery of coil and trapped between the coil loops). D, DSA image obtained during the procedure shows the successful deployment of a LEO Baby stent (arrows) into the parent artery (PICA-vertebral artery). E, Immediate postprocedural DSA demonstrates the near-total occlusion of the aneurysm, with minimal filling in the neck. F, Six-month follow-up DSA shows complete occlusion of the aneurysm and a moderate degree of in-stent stenosis.

\section{Follow-Up}

Angiographic follow-ups were performed in 77 patients $(96.2 \%)$. The mean length of follow-up was $7.2 \pm 3.8$ months (range, 3-22 months). The final angiographic follow-up was performed by using DSA in 64 cases (83.2\%) and MR angiography in 13 patients $(16.8 \%)$. The final follow-up angiograms revealed a class 1 occlusion in 66 patients $(85.7 \%)$, class 2 in 9 patients (11.7\%), and class 3 in 2 patients $(2.6 \%)$. The follow-up of 16 aneurysms with a partial immediate occlusion (Raymond class 2 or 3 ) revealed 9 aneurysms (56.2\%) with an improvement in the Raymond class (progressive occlusion) (Fig 5). Five aneurysms (6.5\%) showed recanalization (deterioration of the Raymond class). Four of the 77 aneurysms with follow-up angiography (5.2\%) required retreatment.

Follow-up angiography demonstrated incidental in-stent stenosis in 12 patients (15.6\%). In-stent stenosis was anatomically mild $(<50 \%)$, not flow-limiting, and asymptomatic in all cases. Consequently, no further interventions were performed, but these patients continued clopidogrel instead of aspirin at follow-up.

The final follow-up mRS score was between 0 and 2 in 71 of 77 patients with a clinical follow-up. The final clinical follow-up in 2 patients with a 0 preprocedural mRS score revealed an mRS score of 3. The mRS score of the patient with a preprocedural mRS score of 3 did not change during follow-up.

\section{DISCUSSION}

The first self-expandable stent was developed in 2002 for the treatment of wide-neck intracranial aneurysms. ${ }^{5,10}$ Several other selfexpandable stents have been introduced since 2002, and SAC has become a widely applied method for the treatment of wide-neck intracranial aneurysms. ${ }^{11}$ Self-expandable stents dedicated to intracranial use have enabled endovascular treatment in otherwise uncoilable aneurysms by providing a scaffold during coiling.

Until recently, the deployment of self-expandable stents necessitated the catheterization of the parent artery with 0.021 - to 0027-inch microcatheters. Low-profile, self-expandable stents (LEO Baby and LVIS Jr), deliverable through microcatheters with an internal diameter of 0.0165 inches, were developed in 2011. ${ }^{7,8,12}$ These low-profile stents allow easier catheterization and navigation in small-sized, delicate vessels and enable safer stent placement during the treatment of distal wide-neck aneurysms. Few articles have investigated the safety and efficacy of the treatment of intracranial aneurysms by using low-profile stents. ${ }^{7,8,12-14}$ The current study reports the retrospective experience of 3 university hospitals by using a low-profile stent system, 

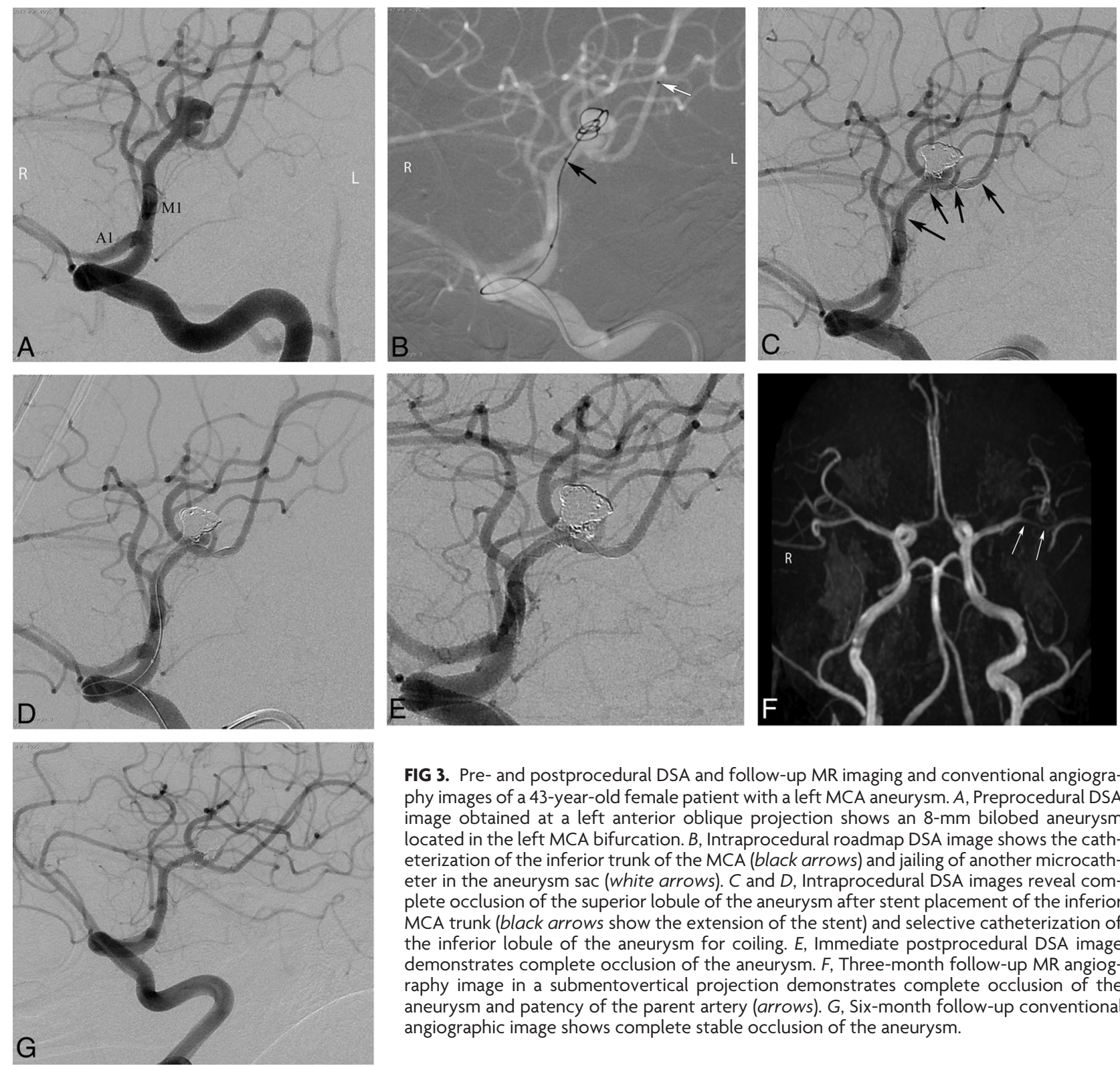

FIG 3. Pre- and postprocedural DSA and follow-up MR imaging and conventional angiography images of a 43-year-old female patient with a left MCA aneurysm. A, Preprocedural DSA image obtained at a left anterior oblique projection shows an 8-mm bilobed aneurysm located in the left MCA bifurcation. B, Intraprocedural roadmap DSA image shows the catheterization of the inferior trunk of the MCA (black arrows) and jailing of another microcatheter in the aneurysm sac (white arrows). $C$ and D, Intraprocedural DSA images reveal complete occlusion of the superior lobule of the aneurysm after stent placement of the inferior MCA trunk (black arrows show the extension of the stent) and selective catheterization of the inferior lobule of the aneurysm for coiling. E, Immediate postprocedural DSA image demonstrates complete occlusion of the aneurysm. F, Three-month follow-up MR angiography image in a submentovertical projection demonstrates complete occlusion of the aneurysm and patency of the parent artery (arrows). G, Six-month follow-up conventional angiographic image shows complete stable occlusion of the aneurysm.

the LEO Baby stent, for SAC treatment of wide-neck intracranial aneurysms.

The LEO Baby stent is a self-expandable, low-profile stent composed of braided mesh nitinol wires. ${ }^{15}$ The LEO Baby stent is not approved by the FDA at the moment. It has received CE mark approval in Europe. LEO Baby stents, similar to LVIS Jr stents, have several advantages over laser-cut stents. First, LEO Baby stents have a sliding-strut design, and this hybrid design allows better wall apposition and scaffolding compared with open and closed cells. Second, LEO Baby stents are resheathable or repositionable up to approximately $95 \%$ of their length. LEO Baby stents have relatively small pore size compared with other selfexpandable intracranial stents. This structural feature provides a tighter mesh, preventing coil protrusion during coiling. However, the small pore size may turn to a disadvantage in cases necessitating the catheterization of an aneurysm through the struts of the LEO Baby stent, though we did not experience such a disadvantage in our practice.
In the present study, the technical success rate of the procedures was high (97.5\%). The flexibility of LEO Baby stents facilitates navigation even in small, tortuous arteries. We used distal-access catheters in cases with tortuous proximal vascular anatomy, which might contribute to successful stent navigation. At the moment, LEO Baby stents are available with diameters of 2.0 and $2.5 \mathrm{~mm}$ and lengths of 12, 18, 25, 30, and 35 $\mathrm{mm}$. LEO Baby stents are labeled for deployment in arteries with diameters between 1.5 and $3.2 \mathrm{~mm}$. Therefore, these are the only self-expandable stents that are labeled for deployment in arteries with diameters of $<2 \mathrm{~mm} .{ }^{15}$ LEO Baby stents were successfully deployed in arteries with diameters as small as 1.3 $\mathrm{mm}$ in our study. Furthermore, the LEO Baby stent could be deployed in branches having a diameter discrepancy of up to $60 \%$. We observed technical complications in 2 cases, but they did not contribute to the angiographic or clinical outcomes of these patients.

Immediate complete occlusion rates after SAC showed vari- 

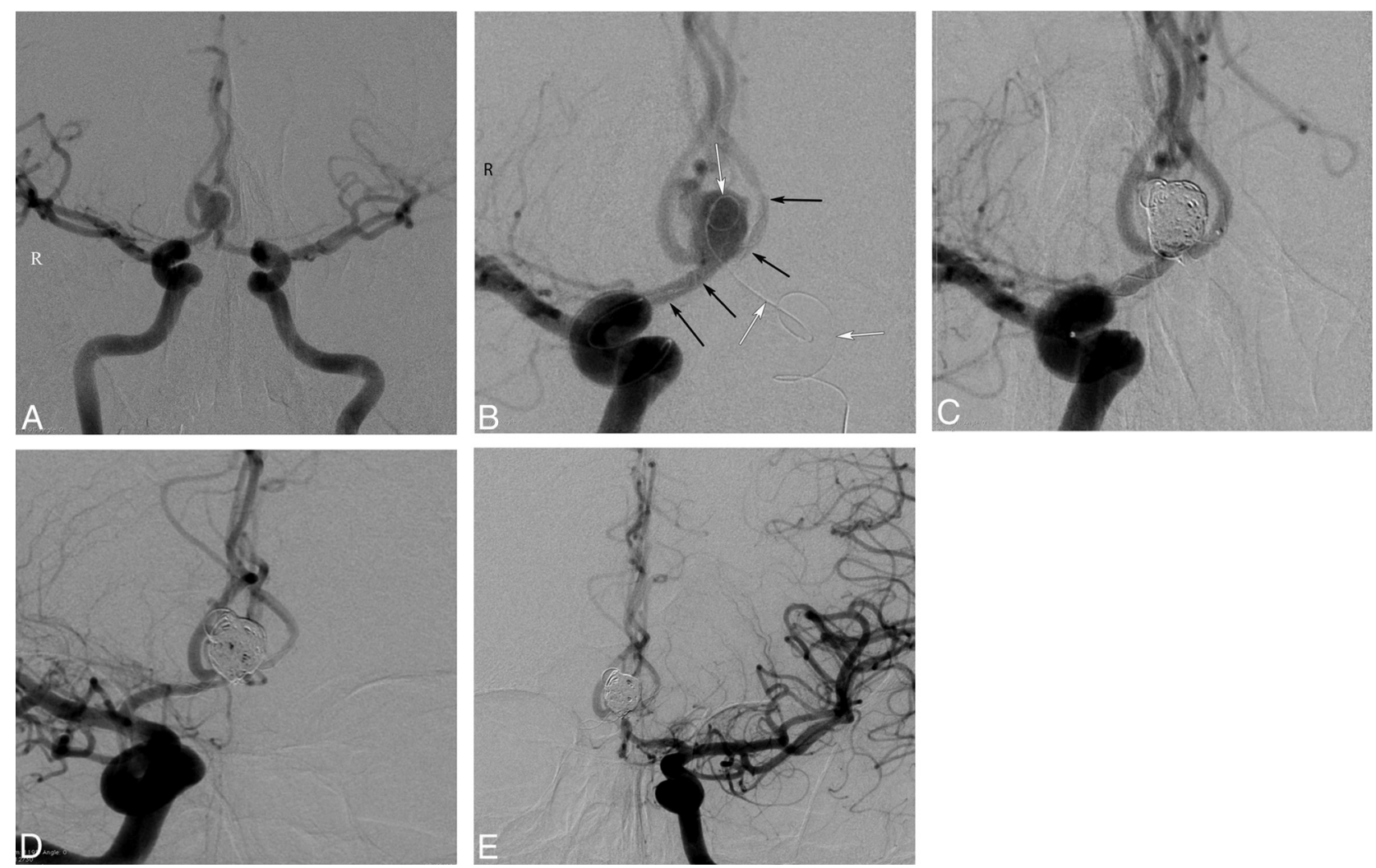

FIG 4. Procedural and follow-up angiographic images of a 42-year-old male patient with an AcomA aneurysm. A, Preprocedural DSA image obtained with bilateral internal carotid artery injections shows the wide-neck 8-mm AcomA aneurysm. $B$, DSA image obtained during the procedure demonstrates deployment of a LEO Baby stent extending from the left A2 to the right Al (black arrows) and a jailed microcatheter in the aneurysm sac (white arrows). C, Postprocedural immediate DSA image shows complete occlusion of the aneurysm. D and $E$, Six-month follow-up DSA images demonstrate complete occlusion of the aneurysm and a mild degree of in-stent stenosis.

ability in the literature but are relatively low compared with coiling study results. ${ }^{6,11}$ A retrospective study by Maldonado et $\mathrm{al}^{16}$ reported that an immediate complete occlusion was achieved in $31.6 \%$ of aneurysms treated with Neuroform stents (Stryker Neurovascular). Lopes et $\mathrm{al}^{17}$ studied the long-term outcomes of 410 patients who were treated with SAC by using Neuroform or Enterprise stents and reported an immediate complete occlusion rate of $43.3 \%$. Piotin et $\mathrm{al}^{6}$ compared the angiographic and clinical results of patients who were treated by using SAC, balloon remodeling, and conventional coiling. The immediate complete occlusion rate in the stented group was $46.3 \%$, but $63.5 \%$ of the aneurysms in the group without stents were completely occluded in immediate postprocedural angiographs. A meta-analysis of the SAC literature by Shapiro et al $^{11}$ revealed an immediate complete occlusion rate of $45 \%$. These relatively poor immediate complete occlusion rates may be because larger and more complex aneurysms are more likely to be treated with stents. Dual antiplatelet therapy in patients with stents might impede the immediate thrombosis of the aneurysm sac, and the difficulty in maneuvering coiling microcatheters between the struts of stents might contribute to the relatively low immediate occlusion rates. Möhlenbruch et $\mathrm{al}^{12}$ recently published a prospective study in a relatively small patient group and reported the safety and efficacy of the LVIS Jr stent, which is one of the available low-profile stents. The immediate complete occlusion rate was $73 \%$. Poncyljusz et al ${ }^{18}$ reported an immediate complete occlusion rate of $85 \%$ by using either LVIS or LVIS Jr stents. The immediate total occlusion rate of $75 \%$ in our study is comparable with the results of previous studies.

Stent placement as a component of SAC treatment promotes the progressive occlusion of partially coiled aneurysms and more effectively impedes recanalization compared with conventional coiling or balloon-remodeling techniques. ${ }^{6,19}$ Stents have some hemodynamic and biologic effects on the parent arteries, which cause a progressive thrombosis of partially coiled aneurysms. Stent deployment across the orifice of an aneurysm redirects blood flow from the aneurysm sac toward the distal parent artery and decreases the hemodynamic stress that contributes to thrombosis of the aneurysmal sac. ${ }^{6,20,21}$ Furthermore, stent-induced neointimal overgrowth leads to healing of the aneurysm neck, promotes progressive thrombosis, and reduces the risk of recanalization. $^{22}$ Gory et $\mathrm{al}^{23}$ reported that $30.9 \%$ of aneurysms with partial immediate occlusion evolved into a complete occlusion during a 6-month follow-up, and the recanalization rate was only $14.5 \%$. The progressive occlusion rate in another study was $69.7 \%$ after a mean follow-up of 8.2 months. ${ }^{24}$ In our study, $56.2 \%$ of aneurysms with a partial immediate occlusion (Raymond classes 2 and 3) showed a progressive occlusion, which is comparable with the results of previous studies, and a complete occlusion (Raymond class 1) rate of $85.7 \%$ was achieved during a mean follow-up of 7.2 months. The filling status deteriorated in only 

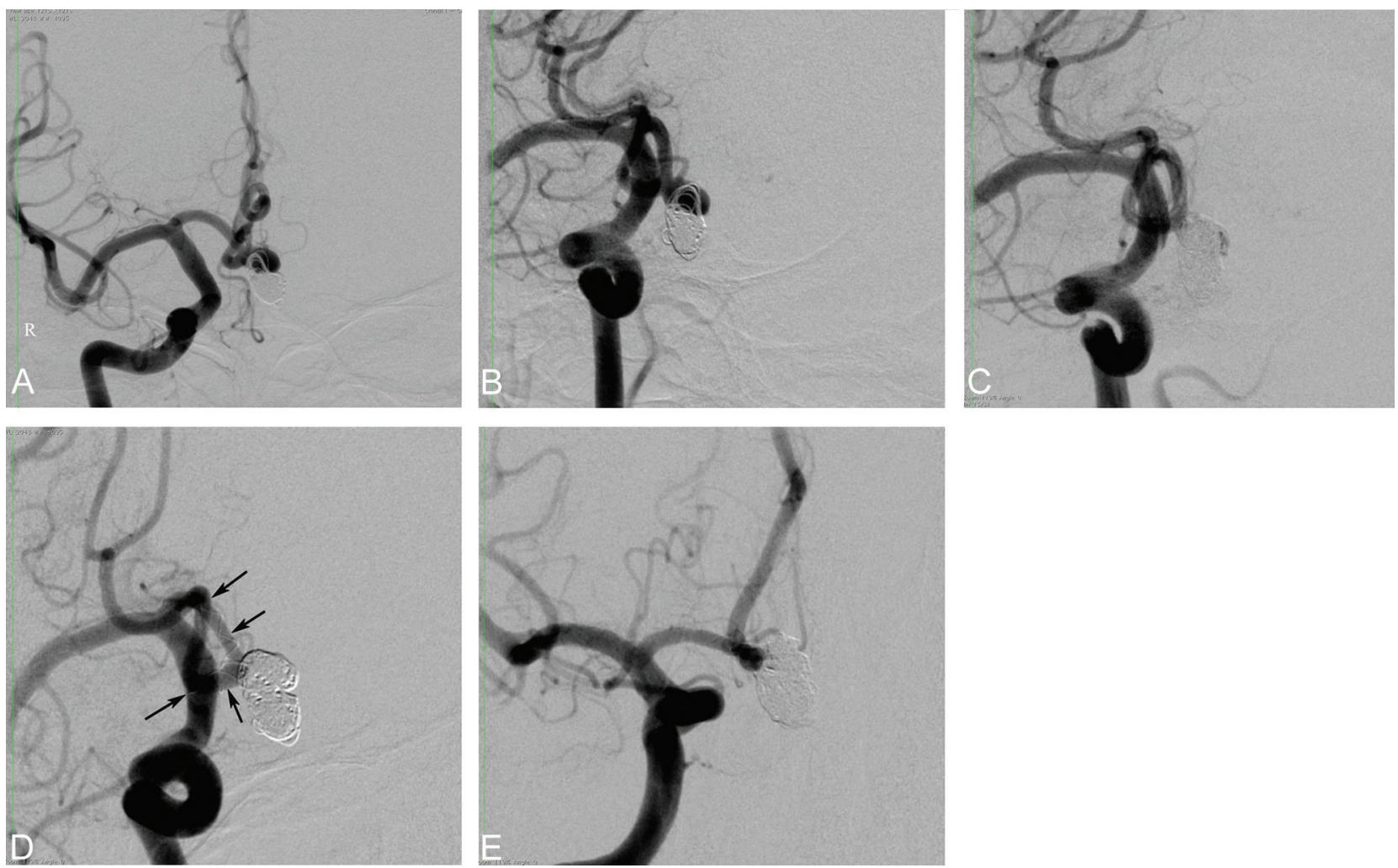

FIG 5. Procedural and follow-up angiographic images of a 53-year-old male patient with an AcomA aneurysm. $A$ and $B$, Preprocedural DSA images show the recanalized AcomA aneurysm that was previously coiled. C, Postprocedural immediate DSA image reveals a partial filling of the aneurysm sac (Raymond class 3). D and E, Nine-month follow-up DSA images demonstrate complete occlusion (Raymond class 1 ) of the aneurysm as a result of progressive thrombosis during the follow-up period (arrows show the extension of the stent).

$6.5 \%$ of aneurysms, and $5.2 \%$ of aneurysms required retreatment during follow-up.

Previous computational fluid dynamics studies demonstrated that stent placement caused a reduction of flow velocity in the aneurysm, and the flow-diversion effect was associated with the lower porosity of stents. ${ }^{25}$ Enhanced flow-diversion capacity promotes the progressive thrombosis of the aneurysm and lowers the risk of recanalization. ${ }^{25,26}$ LEO Baby stents have a relatively high surface coverage ratio of approximately $18 \%$ and a small pore size, which create a relatively high flow-diversion capacity. ${ }^{27}$ Therefore, the flow-diversion capacity of LEO Baby stents might contribute to the development of progressive thrombosis in partially coiled aneurysms by acting as a flow diverter. In support of this hypothesis, the successful treatment of dissecting wideneck aneurysms by using telescopic implantation of LEO Baby stents without coiling was reported. ${ }^{10}$ The favorable midterm angiographic outcomes of this study suggest that LEO Baby SAC is a durable treatment for wide-neck intracranial aneurysms.

The overall complication rate, including asymptomatic minor events, was $11.3 \%$ in our study, which is consistent with that in previous studies. The procedure-related complication rate in a study of the Neuroform SAC of unruptured aneurysms was $12.1 \% .{ }^{28}$ A metaanalysis of the SAC literature conducted by Shapiro et $\mathrm{al}^{11}$ revealed procedure-related complications in 19\% of patients. Most of these complications in the current study remained asymptomatic, and only $3.8 \%$ of the patients had a permanent morbidity. Furthermore, the deficits in all patients with complications were mild to moderate, with mRS scores of $<3$ at the last follow-up, and there was no mortality. Clajus et $\mathrm{al}^{29}$ reported a morbidity of $3.9 \%$ and mortality of $2.9 \%$ for SAC treatment using Solitaire stents (Covidien, Irvine, California). Fiorella et $\mathrm{al}^{30}$ reported that the rates of major ischemic stroke and neurovascular mortality were $8.8 \%$ and $2.8 \%$, respectively, by using Neuroform SAC in 284 patients. A meta-analysis by Naggara et $\mathrm{al}^{31}$ showed that endovascular treatment of unruptured aneurysms was performed with a mortality rate of $1.2 \%$ and a morbidity rate of $4.8 \%$. The morbidity and mortality rates of the current study are comparable with those reported in previous studies. The favorable clinical outcomes of patients in the current study suggest that LEO Baby SAC is a safe endovascular procedure for the treatment of wide-neck intracranial aneurysms.

In-stent stenosis is a previously defined late complication of stent-placement procedures. Fiorella et $\mathrm{al}^{32}$ reported that $5.8 \%$ of patients developed in-stent stenosis following the Neuroform SAC procedure. Another study reported that $3.4 \%$ of patients developed in-stent stenosis after Enterprise SAC on long-term follow-up. ${ }^{21}$ The in-stent stenosis incidence in the current study was quite high compared with those reported in previous studies. However, in-stent stenosis did not cause any symptoms or neurologic findings in our patients. The relatively high rate of progressive occlusion and the flow-diversion capacity of the LEO Baby stent suggest that in-stent stenosis is a consequence of the endothelial healing process that is induced by the stent. $^{30}$

There are some limitations to the current study: First, it was a nonrandomized retrospective study. Therefore, there was no 
control group of potential alternative endovascular treatment methods. Second, follow-up angiography could not be performed in $3.7 \%$ of the patients until after the submission of this article.

\section{CONCLUSIONS}

We reported the results of the largest series of SAC by using a lowprofile stent system, LEO Baby, for the treatment of wide-neck intracranial aneurysms. This study found that a high rate of complete aneurysm occlusion could be achieved by using LEO Baby SAC during the midterm, with an acceptable morbidity rate compared with the results of previous SAC studies. Although in-stent stenosis after LEO Baby SAC is a relatively common phenomenon, it appeared to remain asymptomatic during a midterm follow-up. The coiling of wide-neck intracranial aneurysms with the assistance of low-profile stents is a promising endovascular method.

\section{REFERENCES}

1. Molyneux AJ, Kerr RS, Yu LM, et al; International Subarachnoid Aneurysm Trial (ISAT) Collaborative Group. International Subarachnoid Aneurysm Trial (ISAT) of neurosurgical clipping versus endovascular coiling in 2143 patients with ruptured intracranial aneurysms: a randomised comparison of effects on survival, dependency, seizures, rebleeding, subgroups, and aneurysm occlusion. Lancet 2005;366:809-17

2. Crobeddu E, Lanzino G, Kallmes DF, et al. Review of 2 decades of aneurysm-recurrence literature, part 2: managing recurrence after endovascular coiling. AJNR Am J Neuroradiol 2013;34:481-85

3. Roy D. Aneurysm recurrence after endovascular treatment [in English, French]. Neurochirurgie 2012;58:97-102

4. Higashida RT, Smith W, Gress D, et al. Intravascular stent and endovascular coil placement for a ruptured fusiform aneurysm of the basilar artery: case report and review of the literature. J Neurosurg 1997;87:944-49

5. Akpek S, Arat A, Morsi H, et al. Self-expandable stent-assisted coiling of wide-necked intracranial aneurysms: a single-center experience. AJNR Am J Neuroradiol 2005;26:1223-31

6. Piotin M, Blanc R, Spelle L, et al. Stent-assisted coiling of intracranial aneurysms: clinical and angiographic results in 216 consecutive aneurysms. Stroke 2010;41:110-15

7. Behme D, Weber A, Kowoll A, et al. Low-profile Visualized Intraluminal Support device (LVIS Jr) as a novel tool in the treatment of wide-necked intracranial aneurysms: initial experience in 32 cases. J Neurointerv Surg 2015;7:281-85

8. Akmangit I, Aydin K, Sencer S, et al. Dual stenting using low-profile LEO baby stents for the endovascular management of challenging intracranial aneurysms. AJNR Am J Neuroradiol 2015;36:323-29

9. Roy D, Milot G, Raymond J. Endovascular treatment of unruptured aneurysms. Stroke 2001;32:1998-2004

10. Wanke I, Doerfler A, Schoch B, et al. Treatment of wide-necked intracranial aneurysms with a self-expanding stent system: initial clinical experience. AJNR Am J Neuroradiol 2003;24:1192-99

11. Shapiro M, Becske T, Sahlein D, et al. Stent-supported aneurysm coiling: a literature survey of treatment and follow-up. AJNR Am J Neuroradiol 2012;33:159-63

12. Möhlenbruch M, Herweh C, Behrens L, et al. The LVIS Jr. microstent to assist coil embolization of wide-neck intracranial aneurysms: clinical study to assess safety and efficacy. Neuroradiology 2014; 56:389-95

13. Cohen JE, Melamed I, Itshayek E. X-microstenting and transmesh coiling in the management of wide-necked tent-like anterior communicating artery aneurysms. J Clin Neurosci 2014;21:664-67
14. Conrad MD, Brasiliense LB, Richie AN, et al. Y stenting assisted coiling using a new low profile visible intraluminal support device for wide necked basilar tip aneurysms: a technical report. J Neurointerv Surg 2014;6:296-300

15. LEO + \& LEO + Baby [brochure]. Seoul: Mayabee Design Studio, Balt Extrusion; 2013

16. Maldonado IL, Machi P, Costalat V, et al. Neuroform stent-assisted coiling of unruptured intracranial aneurysms: short- and midterm results from a single-center experience with 68 patients. AJNR AmJ Neuroradiol 2011;32:131-36

17. Lopes DK, Johnson AK, Kellogg RG, et al. Long-term radiographic results of stent-assisted embolization of cerebral aneurysms. $\mathrm{Neu}$ rosurgery 2014;74:286-91

18. Poncyljusz W, Biliński P, Safranow K, et al. The LVIS/LVIS Jr. stents in the treatment of wide-neck intracranial aneurysms: multicentre registry. J Neurointerv Surg 2014 May 14. [Epub ahead of print]

19. Chalouhi N, Starke RM, Koltz MT, et al. Stent-assisted coiling versus balloon remodeling of wide-neck aneurysms: comparison of angiographic outcomes. AJNR Am J Neuroradiol 2013;34: 1987-92

20. Cantón G, Levy DI, Lasheras JC. Hemodynamic changes due to stent placement in bifurcating intracranial aneurysms. J Neurosurg 2005;103:146-55

21. Fargen KM, Hoh BL, Welch BG, et al. Long-term results of Enterprise stent-assisted coiling of cerebral aneurysms. Neurosurgery 2012;71:239-44

22. Lopes D, Sani S. Histological postmortem study of an internal carotid artery aneurysm treated with the Neuroform stent. Neurosurgery 2005;56:E416; discussion E416

23. Gory B, Klisch J, Bonafé A, et al. Solitaire AB stent-assisted coiling of wide-necked intracranial aneurysms: mid-term results from the SOLARE study. Neurosurgery 2014;75:215-19; discussion 219

24. Peng T, Qian Z, Liu A, et al. Progressive occlusion of Enterprise stent-assisted coiling of ruptured wide-necked intracranial aneurysms and related factors on angiographic follow-up: a singlecenter experience with 468 patients. PLoS One 2014;9:e92407

25. Kono $\mathrm{K}$, Terada $\mathrm{T}$. Hemodynamics of $\mathbf{8}$ different configurations of stenting for bifurcation aneurysms. AJNR Am J Neuroradiol 2013;34:1980-86

26. Chalouhi N, Jabbour P, Singhal S, et al. Stent-assisted coiling of intracranial aneurysms: predictors of complications, recanalization, and outcome in 508 cases. Stroke 2013;44:1348-53

27. Spiotta AM, Miranpuri A, Chaudry MI, et al. Combined balloon stent technique with the Scepter $C$ balloon and low-profile visualized intraluminal stent for the treatment of intracranial aneurysms. J Neurointerv Surg 2013;5(suppl 3):iii79-iii82

28. Gentric JC, Biondi A, Piotin M, et al. Safety and efficacy of Neuroform for treatment of intracranial aneurysms: a prospective, consecutive, French multicentric study. AJNR Am J Neuroradiol 2013;34:1203-08

29. Clajus C, Sychra V, Strasilla C, et al. Stent-assisted coil embolization of intracranial aneurysms using Solitaire $\mathrm{AB}$ neurovascular remodeling device: initial and mid-term follow-up results. Neuroradiology 2013;55:629-38

30. Fiorella D, Albuquerque FC, Woo H, et al. Neuroform stent assisted aneurysm treatment: evolving treatment strategies, complications and results of long term follow-up. J Neurointerv Surg 2010;2:16-22

31. Naggara ON, Lecler A, Oppenheim C, et al. Endovascular treatment of intracranial unruptured aneurysms: a systematic review of the literature on safety with emphasis on subgroup analyses. Radiology 2012;263:828-35

32. Fiorella D, Albuquerque FC, Woo H, et al. Neuroform in-stent stenosis: incidence, natural history, and treatment strategies. $\mathrm{Neu}$ rosurgery 2006;59:34-42; discussion 34-42 